\title{
A Comparative Study of Portmanteau Tests for Univariate Time Series Models
}

\author{
Sohail Chand \\ Institute of Statistics \\ University of the Punjab \\ Lahore-Pakistan \\ E-mail: sohail@stat.pu.edu.pk \\ Shahid Kamal \\ Institute of Statistics \\ University of the Punjab \\ Lahore-Pakistan \\ E-mail: director@stat.pu.edu.pk
}

\begin{abstract}
Time series model diagnostic checking is the most important stage of time series model building. In this paper the comparison among several suggested diagnostic tests has been made using the simulation time series data.
\end{abstract}

\section{Introduction}

Until the advent of powerful and accessible computing methods, the experimenter was often confronted with a difficult choice. Either describes an accurate model of a phenomenon, which would usually preclude the computation of explicit answers, or choose a standard model which would allow this computation, but may not be a close representation of a realistic model. This dilemma is present in many branches of statistical applications, for example, in electrical engineering, aeronautics, biology, networks, economics, and astronomy. To use realistic models, the researches in these disciplines have often developed original approaches for model fitting that are customized for their own problems.

Model building is an important part of time series analysis (Chatfield; 2004). The task facing the modern time series is to develop reasonably simple models capable of forecasting, interpreting, and testing hypothesis concerning its field of application (Enders; 2004).

Time series model building is considered as a three-stage iterative procedure based on

- Identification

- Estimation

- Diagnostic checking.

Diagnostic checking is to check the fitted model in its relation to the data with intent to reveal inadequacies and so to achieve the model improvement (Box et. al. 1994). The object of the diagnostic checking stage is not merely to determine 
whether there is evidence of lack of fit but also to suggest ways in which model may be modified when this is necessary.

Two basic methods

1) Overfitting

2) Diagnostic Checks applied to residuals

are suggested.

If the parameters are known exactly, the stochastic process underlying a sample time series can be computed directly from the observations; when this calculation is made with estimates substituted for the true parameter values, the resulting sequence is referred as the residuals, which can be regarded as estimates of the errors. If the appropriate model has been chosen, there will be zero autocorrelation in the errors and introduced a useful tool for testing the adequacy of autoregressive moving average (ARMA) models (Box and Pierce; 1970).

\section{Methods and Material}

Box and Pierce (1970) suggested a statistic to test the adequacy of the fitted time series model given as

$$
Q(r)=n(n+2) \sum_{k=1}^{m} \frac{r_{k}^{2}}{n-k}
$$

would for large $n$ be distributed as $\chi^{2}$ with $m$ degrees of freedom; or as further approximation,

$$
Q(r)=n \sum_{k=1}^{m} r_{k}^{2} \sim \chi_{m}^{2}
$$

Furthermore, they showed that when the $p+q$ parameters of an appropriate model are estimated and the $\hat{r}_{k}$ 's replace the $r_{k}{ }^{\prime} s$, then

$$
Q(\hat{r})=n \sum_{k=1}^{m} \hat{r}_{k}^{2} \sim \chi_{m-p-q}^{2}
$$

Davies, Triggs and Newbold (1977) verified that the distribution of $Q(\hat{r})$ can deviate from $\chi_{m-p-q}^{2}$ and showed that the true significance levels are likely to be much lower than predicted by asymptotic theory.

Ljung and Box (1978) proposed a modified version of the test suggested by Box and Pierce (1970).

$$
\tilde{Q}(\hat{r})=n(n+2) \sum_{k=1}^{m} \frac{\hat{r}_{k}^{2}}{n-k}
$$

They compared their modified test with the test suggested by Box and Pirece (1970) with the help of numerical calculations that the modified test provides a substantially improved approximation to $\chi^{2}$ distribution that should be adequate for most practical purposes. Ljung (1986) examined the properties of the test 
suggested by Box and Pierce (1970) for various choices of $m$ and also suggested a modification which allows the use of small values of $\mathrm{m}$ to result in amore powerful test. He also discussed the Lagrange multiplier statistic (Godfrey, 1979) and a test statistic examined by Newbold (1980).

Monti (1994) proposed a test based on the sum of squared residual partial autocorrelations without noticing Durbin (1980) and also studied the small sample performance of suggested test statistic through a Monte Carlo experiment. The development of suggested test was based on the fact, if the error is a white noise process, one should expect the residual partial autocorrelations to be not significantly different form zero. Therefore an adequacy of fit test can be based on the statistic

$$
\tilde{Q}(\hat{\pi})=n(n+2) \sum_{k=1}^{m} \frac{\hat{\pi}_{k}^{2}}{n-k} \sim \chi_{m-p-q}^{2}
$$

Kwan and Wu (1997) investigated the fine sample performance of Monti's test, paying special attention to its estimated sizes and empirical powers. With the help of simulation they indicated that the test size can be affected by the choice of the number of residual partial autocorrelations, $m$, and the empirical powers of the Monti and the Ljung-Box tests are similar in the cases of both seasonal and non-seasonal data if $\mathrm{m}$ is properly chosen.

\section{Results and Discussion}

We compared the performances of Box-Ljung test and Monti's test under the different alternative hypothesis using Monte Carlo experiment to generate the Gaussian series of 100 observations using STATA software.

Table-1 p-values for Ljung-Box test and Monti's test for AR(1) process

\begin{tabular}{|c|c|c|c|c|c|c|c|c|}
\hline & \multicolumn{6}{|c|}{$\alpha=0.05$} \\
\hline & \multicolumn{2}{|c|}{$\mathrm{m}=7$} & \multicolumn{2}{|c|}{$\mathrm{m}=10$} & \multicolumn{2}{c|}{$\mathrm{m}=15$} & \multicolumn{2}{c|}{$\mathrm{m}=20$} \\
\hline$\varphi$ & $\mathrm{Q}(\mathrm{r})$ & $\mathrm{Q}(\pi)$ & $\mathrm{Q}(\mathrm{r})$ & $\mathrm{Q}(\pi)$ & $\mathrm{Q}(\mathrm{r})$ & $\mathrm{Q}(\pi)$ & $\mathrm{Q}(\mathrm{r})$ & $\mathrm{Q}(\pi)$ \\
\hline 0.1 & 0.047 & 0.046 & 0.046 & 0.048 & 0.049 & 0.047 & 0.055 & 0.043 \\
\hline 0.4 & 0.042 & 0.046 & 0.044 & 0.047 & 0.051 & 0.048 & 0.057 & 0.048 \\
\hline 0.7 & 0.047 & 0.044 & 0.047 & 0.043 & 0.053 & 0.043 & 0.058 & 0.041 \\
\hline 0.9 & 0.049 & 0.056 & 0.062 & 0.055 & 0.064 & 0.052 & 0.074 & 0.048 \\
\hline
\end{tabular}

Table-2 p-values for Ljung-Box test and Monti's test for MA(1) process

\begin{tabular}{|c|c|c|c|c|c|c|c|c|}
\hline \multicolumn{1}{|c||}{} & \multicolumn{7}{c|}{$\alpha=0.05$} \\
\hline & \multicolumn{2}{|c|}{$\mathrm{m}=7$} & \multicolumn{2}{c|}{$\mathrm{m}=10$} & \multicolumn{2}{c|}{$\mathrm{m}=15$} & \multicolumn{2}{c|}{$\mathrm{m}=20$} \\
\hline$\varphi$ & $\mathrm{Q}(\mathrm{r})$ & $\mathrm{Q}(\pi)$ & $\mathrm{Q}(\mathrm{r})$ & $\mathrm{Q}(\pi)$ & $\mathrm{Q}(\mathrm{r})$ & $\mathrm{Q}(\pi)$ & $\mathrm{Q}(\mathrm{r})$ & $\mathrm{Q}(\pi)$ \\
\hline 0.1 & 0.045 & 0.049 & 0.046 & 0.048 & 0.050 & 0.049 & 0.058 & 0.049 \\
\hline 0.4 & 0.041 & 0.050 & 0.051 & 0.050 & 0.056 & 0.048 & 0.064 & 0.048 \\
\hline 0.7 & 0.048 & 0.047 & 0.049 & 0.048 & 0.052 & 0.049 & 0.056 & 0.046 \\
\hline 0.9 & 0.059 & 0.050 & 0.051 & 0.049 & 0.056 & 0.046 & 0.062 & 0.043 \\
\hline
\end{tabular}


Monti's test shows better approximation to chi-square distribution and is at least as good as that of the Ljung-Box statistic.

Monti's test provides stable results over different values of "m".

\section{References}

1. Box, G.E.P et. al. (1994): "Time Series Analysis: Forecasting and Control", 3rd edition, Pearson Education (Singapore) Pte. Ltd.

2. Box, G.E.P. \& Pierce, D.A. (1970). "Distribution of residual autocorrelations in autoregressive-integrated moving average time series models. Journal of the American Statistical Association, Vol. 65 No. 332, 1509-26.

3. Box, G.E.P. and Pierce, D.A. (1970). "Distribution of residual autocorrelations in autoregressive-integrated moving average time series models". Journal of the American Statistical Association, Vol. 65, No. 332, 1509-25.

4. Chatfield, C. (2004): "The Analysis of Time Series: An Introduction", 6th edition, Chapman \& Hall/CRC.

5. Davies, N. and Newbold, P. (1979), "Some power studies of a portmanteau test of time series model specification", Biometrika, Vol. 66, No. 1, 153-5.

6. Davies, N., Triggs, C.M. and Newbold, P. (1977). "Significance levels of the Box-Pierce portmanteau statistics in finite samples", Biometrika, Vol. 64, No. 3, 517-22.

7. Enders, W. (2004): “Applied Econometric Time Series", 2nd edition, John Wiley \& Sons, Inc.

8. Godfrey, L.G. (1979). "Testing the adequacy of a time series model". Biometrika, Vol. 66, No. 1, 67-72.

9. Kwan, A.C.C. and Wu, Y. (1997), "Further results on the finite sample distribution of Monti's portmanteau test for the adequacy of an $\operatorname{ARMA}(p, q)$ model", Biometrika, Vol. 84, No. 3, 733-736.

10. Ljung, G.M. (1986). "Diagnostic testing of univariate time series models". Biometrika, Vol. 73, No. 3, 725-30.

11. Ljung, G.M. and Box, G.E.P. (1978). "On a measure of lack of fit in time series models". Biometrika, Vol. 65, No. 2, 297-303.

12. Monti, A.C. (1994), "A proposal for a residual autocorrelation test in linear models", Biometrika, Vol. 81, No. 4, 776-80.

13. Newbold, P. (1980), "The equivalence of two tests of time series model adequacy", Biometrika, Vol. 67, No. 2, 463-5.

14. Pllana, S. "History of Monte Carlo Method" http://www.geocities.com/CollegePark/Quad/2435/history.html

15. Robert, C.P. and Casella, G. (2004). "Monte Carlo Statistical Methods", 2nd edition, Springer Science+Business Media Inc. 